\title{
Disorder-induced transition from Gaussian to dispersive carrier transport in molecularly doped polymers
}

\author{
Yoshihiko Kanemitsu, Hiroshi Funada, and Yasuaki Masumoto \\ Institute of Physics, University of Tsukuba, Tsukuba, Ibaraki 305, Japan
}

(Received 12 February 1991; accepted for publication 6 May 1991)

\begin{abstract}
Dynamics of hopping charge transport in a polymer matrix doped with two different charge-transport molecules was studied by means of time-of-flight (TOF) photoconductivity measurements. In polymers doped with two molecules of little different ionization potential, the Gaussian (near-rectangular) TOF signal was observed over all compositions and the tail of the Gaussian TOF signal is broadened by a wide distribution of hopping time among molecules due to off-diagonal disorder. On the other hand, in polymers doped with two molecules of different ionization potential, the transition from the Gaussian to the dispersive TOF signal was observed and this is mainly caused by diagonal disorder. It is found that the energetic and spatial distribution of hopping sites play a key role in carrier transport in molecularly doped polymers.
\end{abstract}

Charge transport in amorphous organic photoconductors has been the subject of much theoretical and experimental interest in recent years. ${ }^{1-12} \mathrm{~A}$ variety of systems has been studied: charge-transfer complex, ${ }^{3}$ pure polymers, ${ }^{4,5}$ and molecularly doped polymers (MDPs). ${ }^{6-12}$ Amorphous organic MDPs become particularly attractive because of their importance as photoconductors in electrophotography and the construction of transport theories in amorphous solid. It is recognized that charge transport in MDPs is a hopping process among doped molecules.

Hopping charge transport depends on both the wave function overlap between hopping sites and the energy difference between hopping sites. In amorphous solids, there exist the fluctuation of hopping site energies (diagonal disorder) and the fluctuation of the wave function overlap between hopping sites (off-diagonal disorder). ${ }^{2,4,6,9}$ It is theoretically pointed out that hopping charge transport in MDPs is affected by diagonal and off-diagonal disorder. ${ }^{9}$ However, the effects of disorder in MDPs on hopping charge transport are not clarified experimentally. ${ }^{12}$ On the other hand, very weak van der Waals interaction between molecules leads to very narrow energy bands, although the width of the energy band is inhomogeneously broadened by the disorder matrix. It is therefore considered that in an inert polymer matrix both diagonal and off-diagonal disorder can be controlled by mixing two charge-transport molecules of different ionization potential and different hopping rate. This advantage of MDPs is not easily used in inorganic amorphous systems. In this letter, we report the experimental observation of effects of the spatial and energetic distribution of hopping sites on hopping transport in MDPs by measuring the time-of-flight photocurrent pulse shape and the drift mobility of holes in an inert polymer doped with two different molecules.

Hole-transporting molecules used in the work were PRA [1-phenyl-3-( $p$-diethylaminostyryl)-5-( $p$-diethylaminophenyl)-2-pyrazoline], HD (1-phenyl-1,2,3,4-tetrahydroquinoline-6-carboxyaldehyde-1,1'-diphenyl hydrazone), OX [2-(p-dipropyl-aminophenyl)-4-( $p$-dimethylaminophenyl)-5-(o-chlorophenyl)-1,3-oxazole], DEH ( $p$-di- ethylaminobenzaldehyde-1, 1'-diphenyl hydrazone), MPS [4- $N, N$-bis (4-methylphenyl) amino- $\alpha$-phenylstilbene], PS (4- $N, N$-diphenylamino- $\alpha$-phenylstilbene), and OXD [2,5bis (4'-diethylaminopheny1)-1,3,4-oxadiazole]. The ionization potential, $I_{p}$ (the threshold energy for the ionization of the solid), was determined by using an ultraviolet photoelectron spectroscopy system (RIKEN, AC-1), and $I_{p}$ is shown in Table $\mathrm{I}$. The hole-transporting molecules were dispersed in bisphenol-A-polycarbonate. These in methylene chloride were coated onto the aluminum substrate. The dry film thickness was $12 \mu \mathrm{m}$. The total concentration of two different molecules in solid polycarbonate film was 50 wt $\%$, and the concentration ratio of one molecule to another molecule was varied between 0 and $100 \mathrm{wt} \%$. The semitransparent gold electrode was finally deposited by vacuum evaporation. In the time-of-night (TOF) photoconductivity experiment, the sandwich structure sample was connected in a circuit containing a voltage source and a resistance. The MDPs were excited through the gold electrode by a $0.3 \mathrm{~ns}, 337 \mathrm{~nm}$ pulse from a $\mathrm{N}_{2}$ laser. The current transients were displayed on a digital storage oscilloscope interfaced to a computer. In some experiments, the $0.2 \mu \mathrm{m}$ charge generation films of metal-free phtalocyanine or amorphous selenium were attached to MPDs films. These two structures were excited by a $0.5 \mu \mathrm{s}, 600$ nm pulse from a xenon flash lamp. At high applied field above $1 \times 10^{5} \mathrm{~V} / \mathrm{cm}$, there was no significant TOF waveform difference between single-layer and two-layer structurcs. In Gaussian (near-rectangular) pulse shape, the transit time, $t_{T}$, is determined by the shoulder in the TOF shape. ${ }^{11}$ In dispersive pulse shape, $t_{T}$ is determined from a double $\log$ plot. ${ }^{11}$ The hole drift mobility $\mu$ is given by $\mu=L^{2} / t_{T} V$, where $L$ is the sample thickness and $V$ is the applied voltage.

First, we show TOF results in polymers doped with two molecules of little different ionization potential. Figure 1 summarizes the drift mobility of holes and TOF signal shapes in OXD and PS doped polycarbonate at $2.0 \times 10^{5}$ $\mathrm{V} / \mathrm{cm}$ and $298 \mathrm{~K}$ as a function of the relative weight ratio of OXD to PS. Over all compositions, the Gaussian (near- 
TABLE I. The ionization potential and the hole mobility of charge-transporting molecules.

\begin{tabular}{lcc}
\hline \hline & $I_{p}(\mathrm{eV})$ & $\mu\left(\mathrm{cm}^{2} / \mathrm{V} \mathrm{s}\right)^{\mathrm{a}}$ \\
\hline PRA & 5.0 & $9.1 \times 10^{-7}$ \\
HD & 5.1 & $4.2 \times 10^{-6}$ \\
OX & 5.2 & $7.5 \times 10^{-7}$ \\
DEH & 5.3 & $2.8 \times 10^{-6}$ \\
MPS & 5.5 & $6.1 \times 10^{-5}$ \\
PS & 5.5 & $3.9 \times 10^{-5}$ \\
OXD & 5.5 & $1.7 \times 10^{-7}$ \\
\hline \hline
\end{tabular}

Drift mobility of holes in $50 \mathrm{wt} \%$ doped polycarbonate measured at $2.0 \times 10^{5} \mathrm{~V} / \mathrm{cm}$ and $298 \mathrm{~K}$.

rectangular) TOF shapes were observed. In the OXD-PS system, the hopping rate among PS molecules is much larger than that among OXD molecules, because the mobility of holes in PS doped polycarbonate is two orders of magnitude more than that in OXD doped polycarbonate as shown in Table I. As the OXD concentration increases, the drift mobility of holes decreases and the wave function overlap among PS-OXD and OXD-OXD molecules increase. Since the hopping rate among OXD-PS pairs is larger than that among OXD-OXD pairs, the hopping among OXD-PS molecules dominates the charge transport in the OXD-PS mixed doped system. The drift mobility in the OXD-PS mixed system is estimated by the simple scaling of the mobilities of OXD and PS molecules.

Moreover, in PS and OXD mixed doped systems, the Gaussian TOF signal becomes "dispersive" with decreasing the hole mobility. The "dispersive" Gaussian TOF signal is characterized by the tail broadening of the Gaussian TOF signal, $d$, and $d$ is defined as the following relation:

$$
d=\Delta t_{T} / t_{T}=\left(t_{1 / 2}-t_{T}\right) / t_{T},
$$

where $t_{1 / 2}$ is the time when the photocurrent drops to onehalf of the value at $t_{T}$. In Fig. 1, $d$ is plotted as a function

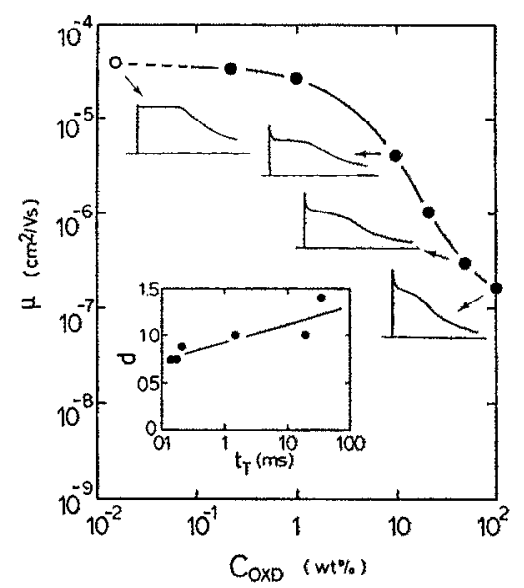

FIG. 1. Hole drift mobility and typical time-of-flight photocurrent pulse shapes in the PS-OXD system at $2.0 \times 10^{5} \mathrm{~V} / \mathrm{cm}$ and $298 \mathrm{~K}$ as a function of relative OXD concentration, $C_{\mathrm{OXD}}$. The open circle denotes the drift mobility of holes at 0 wt \% OXD. There is little ionization potential difference between PS and OXD molecules. The inset shows the tail broadening of the Gaussian time-of-flight signal, $d$ vs the transit time $t_{r}$ of the transit time in the PS-OXD system. The conventional Gaussian nature of the carrier packet moving through the material shows that the ratio of diffusive spread to displacement is given by, $\Delta x / x=(k T)$ $e E)(2 D t)^{-1 / 2}$, where $D$ is the carrier diffusion constant. Using the Einstein relation, $D=(k T / e) \mu$, this relation yields a relative standard deviation of the carrier at the transit time,

$$
\Delta t_{T} / t_{T}=(2 k T / e V)^{1 / 2} .
$$

According to the conventional Gaussian model, $\Delta t_{T} / t_{T}$ is therefore independent of the transit time. However, the inset of Fig. 1 shows that the tail broadening increases with increasing transit time. A theoretical work ${ }^{9}$ suggests that superimposing off-diagonal disorder to the array of energetically ordered hopping sites increases the carrier diffusivity. In the OXD-PS system, the fluctuation of charge transfer rate among hopping sites and the increase in diffusional route due to the mixture of two molecules affect the time-dependent tail-broadening of the Gaussian signals.

Next, we discuss charge transport in polymer matrices doped with two molecules of different ionization potential. If the ionization potential difference between doped molecules is larger than $0.2 \mathrm{eV},{ }^{13}$ the dramatic transition between the Gaussian and featureless dispersive TOF signals was observed as has been reported before. ${ }^{14}$ As an example, Fig. 2(a) shows the hole mobility and TOF signals in the PS-DEH mixed doped system at $2.0 \times 10^{5} \mathrm{~V} / \mathrm{cm}$ and 298 $\mathrm{K}$. It is noted from Fig. 2 that at low DEH concentrations, the TOF signal becomes dispersive and the drift mobility of holes decreases with increasing DEH concentration. Since the ionization potential of the DEH molecule is lower than that of the PS molecule, the DEH molecule acts as a trap for holes: carriers occasionally become localized on DEH molecules. At low DEH concentrations, the wave function overlap among DEH molecules is so small that holes localized at DEH molecules are excited thermally to PS molecules for charge transport. The energy distribution of hopping sites in the mixed doped system causes dispersive transport. On the other hand, Fig. 2 shows that at DEH concentrations higher than $10 \mathrm{wt} \%$, the charge transport is determined only by the hopping among DEH molecules and the TOF signal is Gaussian again. At sufficient high DEH concentrations, the overlap among the DEH molecules becomes large, and so that the DEH-DEH hopping begins to compete with the hopping among PS-PS and PS-DEH molecules: the drift mobility depends on the DEH concentration only and the TOF signal becomes the Gaussian type.

Figure 2(b) shows that the activation energy for hole transport at $2.0 \times 10^{5} \mathrm{~V} / \mathrm{cm}, \Delta$, as a function of the relative DEH concentration in the PS and DEH mixed doped system. The temperature dependence of the drift mobility of holes is usually given by the Arrhenius law: $\mu \propto \exp (-\Delta /$ $k T$ ). On the other hand, Bässler et al. ${ }^{5}$ predicted that the temperature dependence of the mobility is given by $\mu$ $\propto \exp \left[-\left(T_{1} / T\right)^{2}\right]$, where $T_{1}$ is a constant. However, we cannot distinguish whether $T^{-1}$ or $T^{-2}$ is a better descrip- 


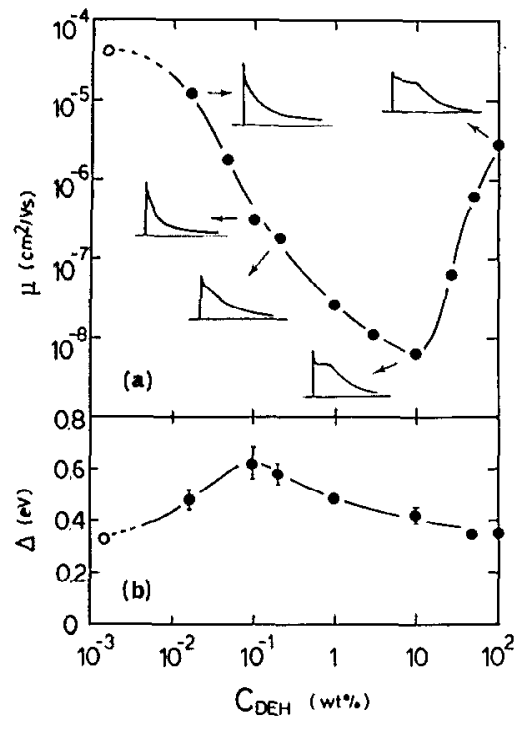

FIG. 2. (a) Hole drift mobility and typical time-of-flight photocurrent pulse shapes in the PS-DEH system at $2.0 \times 10^{5} \mathrm{~V} / \mathrm{cm}$ and $298 \mathrm{~K}$ as a function of relative $\mathrm{DEH}$ concentration, $C_{\mathrm{DEH}}$. The open circle denotes the drift mobility of holes at $0 \mathrm{wt} \% \mathrm{DEH}$. The ionization potential difference between PS and OXD molecules is about $0.2 \mathrm{eV}$. (b) Activation energy for the hole mobility in the PS-DEH system at $2.0 \times 10^{5}$ $\mathrm{V} / \mathrm{cm} . \Delta$, as a function of relative $\mathrm{DEH}$ concentration, $C_{\mathrm{DEH}}$.

tion. In this work the activation energy is determined by the Arrhenius plot. The activation energy for the mobility characterizes the hopping among molecules. It is seen from Fig. 2 that the activation energy for the hopping among molecules increases at the range of dispersive TOF shape. The activation energy reflects the TOF pulse shape rather than the magnitude of the mobility. In trap-controlled hopping, the charge transport is limited by the hopping from the low-trap state to the high-mobile state. The activation energy characterizes the energy depth of trap states. ${ }^{15}$ According to this model, the activation energy of hole mobility in the PS-DEH system $\Delta_{P-D}$ is given by

$$
\Delta_{P-D} \simeq \Delta_{D-D}+\Delta I_{p},
$$

where $\Delta_{D-D}$ is the activation energy of the hopping among DEH molecules, and $\Delta I_{p}$ is the ionization potential difference between DEH and PS molecules. Since the activation energy $\Delta_{D-D}$ is independent of the concentration of DEH molecules in polycarbonate, ${ }^{10}$ one expects that in the mixed doped system $\Delta_{P-D}$ does not depend on the DEH concentration. However, the experiments show that $\Delta_{P-D}$ is very sensitive to the concentration of DEH molecules. We believe that the spatial and energetic fluctuation of hopping sites cause the dispersion of carriers and increase the activation energy for charge transport. If the energetic fluctuation of hopping sites ( $\Delta I_{p}$ in this case) is not negligible compared with the activation energy in a polymer doped one molecule, the transition from Gaussian to dispersive TOF signal occurs. The nature of hopping charge transport in MDPs is considered to be clarified by the addition of disorder concept to the conventional trap-controlled ${ }^{14}$ or polaron transport models ${ }^{12}$.

In summary, we have studied the effect of disorder on diffusion and drift of carriers in a polymer matrix doped with two different molecules. The anomalous tail broadening of Gaussian TOF signal is caused by a wide distribution of hopping rate among molecules. The dramatic transition between Gaussian to dispersive TOF signal is caused by the energetic distribution of hopping sites. A wide distribution of hopping time among molecules due to diagonal and off-diagonal disorder leads to dispersion of transiting charge packet and plays a key role in determining the feature of TOF signals.

${ }^{1}$ G. Pfister and H. Scher, Adv. Phys. 27, 747 (1978).

${ }^{2}$ J. Mort and G. Pfister, Electronic Properties of Polymers, edited by J. Mort and G. Pfister (Wiley, New York, 1982), p. 215.

${ }^{3}$ W. G. Gill, J. Appl. Phys. 43, 5033 (1972).

${ }^{4}$ F. C. Bos and D. M. Burland, Phys. Rev. Lett. 58, 152 (1987).

${ }^{5}$ M. A. Abkowitz, M. J. Rice, and M. Stolka, Philos. Mag. B 61, 25 (1990).

${ }^{6}$ H. Bässler, G. Schönherr, M. Abkowitz, and D. M. Pai, Phys. Rev. B 26, 3105 (1982).

${ }^{7}$ M. Stolka, J. F. Yanus, and D. M. Pai, J. Phys. Chem. 88, 4707 (1984).

${ }^{8}$ H. J. Yuh and M. Stolka, Philos. Mag. B 58, 539 (1988).

${ }^{9}$ R. Richert, L. Pautmeier, and H. Bässler, Phys. Rev. Lett. 63, 547 (1989); L. Pautmeier, R. Richert, and H. Bässler, Synth. Met. 37, 271 (1990).

10 J. X. Mack, L. B. Schein, and A. Peled, Phys. Rev, B 39, 7500 (1989).

"I. Y. Kanemitsu and J. Einami, Appl. Phys. Lett. 57, 673 (1990).

${ }^{12}$ L. B. Schein, D. Glatz, and J. C. Scott, Phys. Rev. Lett. 65, 472 (1990).

${ }^{13}$ If the ionization potential difference is about $0.1 \mathrm{eV}$ (for example, the OX-HD system), the TOF signal is Gaussian over all compositions.

${ }^{14}$ H. J. Yuh, D. Abramsohn, and M. Stolka, Philos. Mag. Lett. 55, 277 (1987).

${ }^{15}$ G. Pfister, S. Grammatica, and J. Mort, Phys. Rev. Lett. 37, 1360 (1976). 